\title{
From mesoscale to nanoscale mechanics in single-wall carbon nanotubes
}

\author{
Abraao C. Torres-Diass,b,*, Tiago F.T. Cerqueira ${ }^{\mathrm{a}, \mathrm{c}}$, Wenwen Cui ${ }^{\mathrm{a}}$, Miguel \\ A.L. Marques ${ }^{\mathrm{d}, \mathrm{a}}$, Silvana Botti ${ }^{\mathrm{c}, \mathrm{a}}$, Denis Machon ${ }^{\mathrm{a}}$, Markus A. Hartmann ${ }^{\mathrm{e}}$, \\ Yiwei Sun ${ }^{\mathrm{f}}$, David J. Dunstan ${ }^{\mathrm{f}, *}$, Alfonso San-Miguel ${ }^{\mathrm{a}, * *}$ \\ ${ }^{a}$ Université de Lyon, F-69000 Lyon, France and Institut Lumière Matière, CNRS, UMR \\ 5306, Université Lyon 1, F-69622 Villeurbanne, France \\ ${ }^{b}$ Departamento de Física, Universidade Federal do Ceará, Fortaleza, Ceará, 60455-900 \\ Brazil \\ ${ }^{c}$ Institut für Festkörpertheorie und-optik, Friedrich-Schiller-Universität Jena, \\ Max-Wien-Platz 1, D-07743 Jena, Germany \\ ${ }^{d}$ Institut für Physik, Martin-Luther-Universität Halle-Wittenberg, D-06099 Halle, \\ Germany \\ ${ }^{e}$ Faculty of Physics, University of Vienna, 1090 Vienna, Austria \\ ${ }^{f}$ School of Physics and Astronomy, Queen Mary University of London, London E1 $4 N S$, \\ $U K$
}

\begin{abstract}
The analysis of the collapse of individualized and isolated single-wall carbon nanotubes under high pressure as function of their diameter, $d$, distinguishes their mesoscale and their nanoscale mechanics. The evolution with pressure of the Raman spectra for nine tube chiralities and the theoretical modelling reveal a deviation from the continuum mechanics prediction of a collapse pressure $P_{C} \propto d^{-3}$. Nanotubes show a normalized collapse pressure $P_{N}=$ $P_{C} d^{3}=24 \alpha D\left(1-\beta^{2} / d^{2}\right)$ both in experiment and in very different theoretical models. In this expression $\beta=0.44 \pm 0.04 \mathrm{~nm}$ represents the smallest diameter for a stable freestanding single-wall carbon nanotube and $D$ is the bending stiffness of graphene. From the experimental data $D=1.7 \pm$ $0.2 \mathrm{eV}$. Deviations from the continuum mechanics predictions start to be of
\end{abstract}

\footnotetext{
*Corresponding author

**Principal corresponding author

Email addresses: abraaocefas@gmail.com (Abraao C. Torres-Dias),

d.dunstan@qmul.ac.uk (David J. Dunstan), alfonso.san-miguel@univ-lyon1.fr (Alfonso San-Miguel)
} 
significance for diameters smaller than $\sim 1 \mathrm{~nm}$. The associated reduction of their collapse pressure is attributed to the discretization of the elastic compliances around the circumference of the tubes.

\section{Introduction}

Advances in nanoscience open new possibilities to understand how size and geometrical parameters influence mechanical behaviour. It is always interesting to consider if a difference between mesoscale and nanoscale mechanics is a consequence of size (e.g. quantum effects such as quantum confinement, also the scaling laws that account for the mechanical differences between a crane-fly and an elephant) or a consequence of number: where the continuum approximation assumes very large numbers of atoms but at the nanoscale there are only a few atoms. Here we show that the collapse pressure of nanotubes follows continuum mechanics together with a deviation which can be described as an effect of number, not an effect of size.

Single-wall carbon nanotubes (SWCNT) are particularly remarkable as models predict that their radial cross-section should collapse under high pressure, with a strong dependence on their diameter, $d$. The range of available diameters in carbon nanotubes provides a unique opportunity to explore the validity of theory and models from the mesoscale to the nanoscale. Here we show a remarkable transition from the continuum mechanics to a behavior for the smallest tubes driven by geometrical (atomistic) discretization.

Continuum mechanics predicts the radial collapse pressure to depend on the nanotube diameter $d$ following the Lévy-Carrier form $[1-5] d^{-3}$ developed 
in the context of tubes in steam boilers [6] and oil wells [7]. Also other forms such as $d^{-1}$ have been proposed [8]. Experimental results are quite contradictory. For the most-studied SWNCTs having $d \sim 1.35 \mathrm{~nm}$, some experiments suggest a structural phase transition onset at $\sim 2 \mathrm{GPa}[9,10]$, whilst others suggest a collapse transition pressure $\sim 10-15$ GPa [11-13]. Experimental difficulties in identifying the collapse transition include the use of samples having wide distributions of diameter, of samples having both open and closed carbon nanotubes which allows partial filling with the pressure transmitting medium [14], of samples consisting of bundles of nanotubes [15], and difficulties due to the evolution of the Raman resonances with pressure $[16,17]$. Recent experiments on individualized empty (closed) SWCNT [14] showed excellent agreement with improved simulations [5] at an averaged diameter, but were not used for a quantitative experimental determination of the diameter dependence of the collapse pressure.

A major theoretical difficulty is the uncertainty in the values of the bending stiffness, $D$, and thickness, $h$ of graphene; the Lévy-Carrier result is $P_{C}=24 D / d^{3}$ only for $h=0$. There is a wide diversity of predicted $D$ and $h$ values $[18,19]$ but at present the few experimental determinations of $D$ give values ranging from $1.2 \mathrm{eV}[20]$ to $7.1_{-3.0}^{+4.0} \mathrm{eV}[21]$. The bending stiffness of bilayer graphene has been measured (e.g. $D \sim 20-55 \mathrm{eV}$ [21]) but this is due to the in-plane stiffness of graphene, not to the monolayer bending stiffness. Monolayer cantilevers have also been studied, with $D \sim$ $1-10 \mathrm{keV}[22]$ but this high value is attributed to the ripples or corrugations 
in monolayer graphene, which again invokes the in-plane stiffnesses $c_{11}$ and $c_{12}$ of graphene. Studying the bending stiffness in SWCNTs avoids these issues.

In this paper, we revisit experimental data for Raman radial breathing modes (RBM) from the well-characterised individualized tubes of Ref. 14 to obtain - by putting physical constraints on the fits to the Raman spectra - the collapse pressures of carbon nanotubes having different diameters and chiral indices $(m, n)$. We compare the experimental results with our predictions using elastic-continuum and atomistic models as well as Monte Carlo (MC) and quantum mechanical tight-binding (DFTB) semi-empirical simulations which use parameters from density-functional theory. Results identify the underlying $d^{-3}$ dependence of the collapse pressure. In this way the data from the 1-D nanotubes is exploited to give a fundamental 2-D material parameter, that is a direct and reliable measurement of the graphene bending stiffness modulus, $D=1.7 \pm 0.2 \mathrm{eV}$ in excellent agreement with our DFTB calculations and also with the "typical value" of $1.6 \mathrm{eV}$ reported by Lambin [19].

For small diameters we identify an interesting deviation below the LévyCarrier formula in both experiments and theory. This is evidence for the transition from the continuum model at the mesoscale to the onset of atomistic (i.e. geometric or number, not size) effects at the nanoscale. 


\section{Experiments}

The details of the preparation of individualized carbon nanotubes samples and of the high pressure Raman experiments are given elsewhere [14, 23, 24]. The final sample consists of individualized, mostly empty SWCNTs in $\mathrm{D}_{2} \mathrm{O}$ :DOC solution. Empty and water-filled SWCNTs of the same $(m, n)$ are spectroscopically distinguished through the blue shift of the RBM frequency of the filled SWCNTs. The $\mathrm{D}_{2} \mathrm{O}$ :DOC serves also as the pressure-transmitting medium (PTM) for the high-pressure experiments, which were carried out in a diamond-anvil high-pressure cell [14].

Figure 1 shows some of the Raman spectra collected from the sample during a single pressure run. The RBMs of ten different empty SWCNTs having peak positions at ambient pressure from $176 \mathrm{~cm}^{-1}$ to $298 \mathrm{~cm}^{-1}$ could be distinguished. The peaks could readily be identified [24] as empty tubes with the $(m, n)$ values given in Figure 1. These correspond to diameters $d$ ranging from $0.771 \mathrm{~nm}$ to $1.376 \mathrm{~nm}$. Under pressure, there is progressive blueshift [14], broadening and consequent overlapping of the RBM peaks. Concomitantly, the Raman intensities weaken and quench. Our experimental data analysis focused on the intensities. Fits to some of the spectra are shown in Figure 1; for details see the Supplementary Electronic Material.

The resulting RBM peak area (intensity) evolution is plotted in Figure 2. Following a large loss of intensity from 0 to $\sim 1 \mathrm{GPa}$, all nine RBM peaks in the Raman spectra show a plateau followed by a very clear quenching at different pressures depending on the tube diameter. In Figure 2 the data are 
least-squares fitted by the function $1 / 2\left(I_{0} \exp (-b P)+a\right) \operatorname{erf}\left(w / P_{0}\left(P-P_{0}\right)\right)$ where the error function provides an approximation to a trilinear function describing the plateau and the collapse. Data and fits are divided by $a$ to normalise the plateaux to 1 and $b$ is a fitting parameter. With $w$ as a free fitting parameter, fitting those datasets which have several datapoints in their regions of quenching $((8,3),(7,5),(7,6), 13,4))$, we obtain values for $w$ close to 5 and so we fixed $w=5$ for all datasets.

Comparison of the error function with the recent analytic solution for the progressive collapse of the simple elastic ring under pressure $[2,25]$ and some earlier studies, particularly molecular dynamics (MD), e.g. [26-28], permits the identification of the physical significance of the $w$ parameter (width of the error function). There is a continuous evolution, from the transition at the onset of collapse from circular to oval cross-section at $P_{C}$, until the tube walls come into contact at about $1.5 P_{C}$. It is plausible then to identify the pressure range $P_{C}$ to $1.5 P_{C}$ with the width of the error function, about $0.8 P_{0}$ to $1.2 P_{0}$ for $90 \%$ to $10 \%$ intensity. That is, the RBM quenching tracks the progression of the collapse from first ovalisation at $P_{C}=0.8 P_{0}$ to completion at $\sim 1.5 P_{C}=1.2 P_{0}$.

\section{Modeling}

In the following we model the tube collapse as a function of $(m, n)$ using $\mathrm{MC}$ and DFTB as well as simpler models, taking especial care to examine the progression from the onset of collapse to its completion. For details of 
the modelling, see the Supplementary Electronic Material.

Progression of the nanotube collapse is readily monitored in bifurcation diagrams which show the evolution with pressure of the largest and smallest tube diameter normalized by the initial circular tube diameter [28]. The onset of collapse corresponds then to a bifurcation, and its completion to a value of the smallest diameter close to the graphite interlayer distance. This is done in Figure 3 for $\mathrm{MC}$ and $\mathrm{DFTB}$ as well as for two simple models, the elastic ring model $[2,25]$ and the polygonal model [29]. The latter is derived from the continuum elastic ring model by moving all the distributed compliance to $n$ discrete points. As expected for the large Hooke's Law 22gon in Figure 3, excellent agreement is found with the Hooke's Law elastic ring bifurcation curve.

In the MC simulations presented here SWCNTs were described by a non-reactive many-body potential parametrized by ab initio calculations [30]. The validity of this potential for describing carbon nanostructures was tested in Ref. 31. In the simulations, armchair and zig-zag nanotubes with diameters up to $5.7 \mathrm{~nm}$ were hydrostatically loaded. Some of the collapse pressures were previously reported [29].

Most of the DFTB bifurcation curves show discontinuous jumps to the completely collapsed state as shown in Figure 3 for two $(m, n)$. These discontinuities thus indicate - or over-estimate - the pressures at the end of collapse, $\sim 1.5 P_{C}$. The onset of the collapse is generally not detectable, with a few possible exceptions such as the $(10,10)$ tube in Figure 3, while more or 
less ovalisation occurs even from the lowest pressures. These phenomena are doubtless due to the finite strength of the frozen PTM, which enables it both to apply shear stress to the tubes, and to resist the deformation of collapse. In contrast, the MC results show bifurcations similar in form to the elastic ring, so both onset and completion of collapse are clearly indicated. The MC collapse is a little faster than the simple models, as if the bending stiffness is softening slightly at large curvatures.

Table 1: Parameters for the bending stiffness, $D$, determined from the fits of Figure 4 . Fits to the data give $\beta$ and $D ; \alpha$ is a fixed correction factor (see text). Note that $1 \mathrm{GPanm}^{3}=$ $6.242 \mathrm{eV}$.

\begin{tabular}{|c|c|c|c|}
\hline & $\begin{array}{c}\beta \\
(\mathrm{nm})\end{array}$ & $\alpha$ & $\begin{array}{c}D \\
(\mathrm{eV})\end{array}$ \\
\hline Monte Carlo & $0.48 \pm 0.02$ & 1.5 & $1.8 \pm 0.4$ \\
\hline MD bundles [8] & $0.51 \pm 0.03$ & 1.5 & $1.26 \pm 0.05$ \\
\hline DFTB Ar & $0.46 \pm 0.06$ & 1.5 & $2.34 \pm 0.15$ \\
\hline DFTB $\mathrm{H}_{2} \mathrm{O}$ & $0.45 \pm 0.05$ & 1.5 & $2.33 \pm 0.17$ \\
\hline DFTB bundles [5] & $0.41 \pm 0.01$ & 1.5 & $1.72 \pm 0.04$ \\
\hline Experiment & $0.51 \pm 0.05$ & 1 & $1.7 \pm 0.2$ \\
\hline
\end{tabular}

\section{Discussion}

Our experimental and theoretical results for the collapse pressures are collected and compared in Figure 4. We also compare them with the MD and G-band Raman data reported in the literature et al. [8] and the DFTB results previously reported for bundled nanotubes without PTM [5] (these are given for radii up to only $1.35 \mathrm{~nm}$ since at larger diameters they are dominated by effects of bundling which are outside the scope of this paper). Values for the 
onset of collapse and the completion of collapse are shown by solid and open symbols respectively. On this plot, all pressures are normalised by $d^{3}$ so that the continuum mechanics elastic ring prediction, $P_{C} d^{3}=24 D$, corresponds to a horizontal straight line. This Figure thus focuses on the deviations from $P_{C} \propto d^{-3}$, which are small compared with the enormous range of $P_{C}$ given by the $d^{3}$ factor of 125 across the width of the Figure. The data of Elliott et al. [8] presented in that work as a $P_{C} \propto d^{-1}$ fit follow also the $P_{C} \propto d^{-3}$ asymptotic trend for large $d$. Remarkably, all the data (experimental as well as theoretical) show a reduction in $P_{C} d^{3}$ for smaller tubes, similar to that reported previously for the polygon-model (shown, rescaled, in Figure 4) and for the MC model [29] as well as for DFTB calculations [5]. In the polygon model, the $R^{-3}$ dependence was observed for all $n_{g}$-gons, but with a correction term in $n_{g}$ so that the normalised collapse pressure could be written as $P_{C}^{N} \simeq 24 D\left(1-\beta_{g}^{2} / n_{g}^{2}\right)$ with $\beta_{g} \simeq 5.3$ (here $\beta_{g}$ stands for "geometric $\left.\beta^{\prime \prime}\right)$. That is, it is not the $D R^{-3}$ dependence which is modified, but instead the prefactor which becomes $24\left(1-\beta_{g}^{2} / n_{g}^{2}\right)$

We find empirically that the behavior both for small tubes and for large tubes follows a modification of the Lévy-Carrier formula as $P_{C} d^{3}=24 \alpha D(1-$ $\beta^{2} / d^{2}$ ), allowing the determination of the graphene bending modulus, $D$. In this formula $\alpha$ is a correction factor which has the value 1 or 1.5 for the onset or the end of the collapse pressure, respectively, as already discussed. By fitting this expression to all the data in Figure 4 we obtain the values of $\beta$ and $D$ given in Table 1. A remarkable agreement on $\beta$, describing the 
deviation from the $d^{-3}$ behavior, is found among the different theoretical and experimental data. All the values in Table 1 are consistent with $\beta=$ $0.44 \pm 0.04 \mathrm{~nm}$. That is, in individualized and bundled tubes, $\beta$ is not greatly affected by the details of the interatomic potentials between the tube and the environment (water, argon or other tubes in DFTB; no environment in $\mathrm{MC}$ and MD) but its value is mainly due to the nanotube geometry corresponding to the discretization of the elastic compliance. Consistent with the polygon model, the behaviour may also be written as $P_{C} d^{3}=24 \alpha D\left(1-\beta^{\prime 2} /\left(n^{2}+\right.\right.$ $\left.n m+m^{2}\right)$ ) with the dimensionless $\beta^{\prime}=\beta \pi / a \simeq 5.6$.

It is noteworthy that the value of $\beta \simeq 0.44 \mathrm{~nm}$ coincides with the diameter of the thinnest freestanding SWCNTs reported $(0.43 \mathrm{~nm})[32]$. Within the statistical error bars the effect of $\beta$ on the collapse pressure begins to be important for diameters below $\sim 1 \mathrm{~nm}$, distinguishing the regimes of nanoand meso-scale mechanics. Other works $[33,34]$ used very different physical arguments to explain deviations from the Lévy-Carrier formula proposing a surface energy term due to the surrounding environment. Our observation of a $\beta$ at most only weakly dependent on environment appears not to favor that proposition. A particular case is double-walled carbon nanotubes in which the nanotube is in strong interaction - from growth - with the external tube. In such case, the observation of carbon nanotubes with diameters below $0.43 \mathrm{~nm}$ has been reported [35]. This underlines that our result only applies for unsupported single-walled carbon nanotubes and it is consistent with the observation of higher collapse pressures in double-walled carbon 
nanotubes with respect to the expected one for the corresponding internal tube [36]. We may also note that the maximum collapse pressure given by our modified Lévy-Carrier formula is obtained for tubes having a diameter of $d_{m}=\sqrt{5 / 3} \beta=0.57 \mathrm{~nm}$, which will make a $(6,2)$ chirality the more stable free-standing carbon tube with a collapse pressure onset of $14.3 \mathrm{GPa}$. Our modified Lévy-Carrier formula leads to deviations from predictions of continuous mechanics which are of $10 \%$ for tube diameters of $1.39 \mathrm{~nm}$ and which become of about $20 \%$ for tubes of $1 \mathrm{~nm}$ of diameter.

Turning to the graphene bending modulus, $D$, the DFTB data and the MD data of Ref. 8 display discontinuous transitions to complete collapse, so they monitor the end of the collapse at $P=1.5 P_{C}$, i.e. $\alpha=1.5$. Taking this correction into account, Table I gives the deduced values of $D$ for each method. For the MC simulations, $\alpha=1.5$ as this is the known factor relating the collapse pressure obtained by hydrostatic and radial load, respectively [37]. All theoretical values are seen to fall into the range $1.3-2.4 \mathrm{eV}$, which could be considered to constitute excellent agreement with the experimental value from this work of $1.7 \pm 0.2 \mathrm{eV}$. The $D$ value from the DFTB results on bundles [5] gives an excellent agreement with our experimental data. The larger $D$ values for the DFTB modeling with argon and water are consistent with the solidification and the observed tube-like structure of the first shell of the PTM around the tube which confers an additional mechanical support [38].

These results present interesting theoretical challenges for the future. 
The geometrical effect in small tubes is understood qualitatively but not quantitatively. The physical significance of the nanotube value of $\beta \approx 0.5 \mathrm{~nm}$ requires further investigation, perhaps in relation to the limits of stability at small diameters for free-standing nanotubes. The different methods agree much better on $\beta$ than they do on the value of $D$. This fact suggests that $\beta$ is related not to the details of the interatomic potentials or surface energies but to the geometry of the tubes.

What is clearly determined here is that the onset and completion of the RBM quenching correspond well to the onset at $P_{C}$ and completion at $\sim 1.5 P_{C}$ of the nanotube radial collapse. These data then confirm the underlying Lévy-Carrier $d^{-3}$ dependence of the collapse pressure as in continuum mechanics but the progressive deviation from $d^{-3}$ at smaller diameters due at least in part to the geometrical effect of the atomistic nature of the carbon nanotubes. These results provide the ultimate limits at the mesoscale of the application of the continuum mechanics theory of tube stability under external pressure, a problem first addressed 200 years ago for steam boilers and later for oil wells. Finally a direct and rigorous experimental determination of the bending stiffness of graphene follows, as $D=1.7 \pm 0.2 \mathrm{eV}$.

\section{Acknowledgments}

The experimental work was carried out in collaboration with W. Wenseleers and S. Cambré at the University of Antwerp, Belgium. The computational results presented have been achieved in part using the Vienna Scientific 
Cluster (VSC). DJD is grateful for support from the Region Rhône-Alpes through the programme "Accueil-PRO 2014" and from the iMUST Labex. ACTD, TFTC, WC, MALM, SB, DM and ASM acknowledge support from the French Agence Nationale de la Recherche through contract ANR-11NANO-025 "TRI-CO". ACTD acknowledges postdoctoral grant from Brazilian Ministry of Education (CAPES).

\section{References}

[1] M. Lévy, Mémoire sur un nouveau cas intégrable du probleme de l'élastique et l'une de ses applications, J. Math. Pures Appl. 10 (1884) 5.

[2] P. Djondjorov, V. Vassilev, I. Mladenov, Analytic description and explicit parametrisation of the equilibrium shapes of elastic rings and tubes under uniform hydrostatic pressure, Int. J. Mech. Sci. 53 (2011) $355-364$.

[3] P. Tangney, R. B. Capaz, C. D. Spataru, M. L. Cohen, S. G. Louie, Structural transformations of carbon nanotubes under hydrostatic pressure, Nano Lett. 5 (11) (2005) 2268-2273. doi:10.1021/nl051637p.

[4] M. Hasegawa, K. Nishidate, Radial deformation and stability of singlewall carbon nanotubes under hydrostatic pressure, Phys. Rev. B 74 (2006) 115401. doi:10.1103/PhysRevB.74.115401. 
[5] T. F. T. Cerqueira, S. Botti, A. San-Miguel, M. A. L. Marques, Density-functional tight-binding study of the collapse of carbon nanotubes under hydrostatic pressure, Carbon 69 (2014) 355-360. doi:10.1016/j.carbon.2013.12.036.

[6] W. Fairbairn, On the resistance of tubes to collapse, Philos. Trans. R. Soc. London 148 (1858) 389-413.

[7] W. Clinedinst, A rational expression for the critical collapsing pressure of pipe under external pressure, API Drilling and Production Practices 39 (1939) 383.

[8] J. A. Elliott, J. K. W. Sandler, A. H. Windle, R. J. Young, M. S. P. Shaffer, Collapse of single-wall carbon nanotubes is diameter dependent, Phys. Rev. Lett. 92 (2004) 095501. doi:10.1103/PhysRevLett.92.095501.

[9] U. D. Venkateswaran, A. M. Rao, E. Richter, M. Menon, A. Rinzler, R. E. Smalley, P. C. Eklund, Probing the single-wall carbon nanotube bundle: Raman scattering under high pressure, Phys. Rev. B 59 (1999) 10928. doi:10.1103/PhysRevB.59.10928.

[10] M. J. Peters, L. E. McNeil, J. P. Lu, D. Kahn, Structural phase transition in carbon nanotube bundles under pressure, Phys. Rev. B 61 (2000) 5939. doi:10.1103/PhysRevB.61.5939.

[11] A. Sood, P. Teresdesai, D. Muthu, R. Sen, A. Govindaraj, C. Rao, Pressure behaviour of single wall carbon nanotube bundles 
and fullerenes: A Raman study, Phys. Status Solidi B 215 (1) (1999) 393-401. doi:10.1002/(SICI)1521-3951(199909)215:1;393::AIDPSSB393¡3.0.CO;2-8.

[12] C. Caillier, D. Machon, A. San-Miguel, R. Arenal, G. Montagnac, H. Cardon, M. Kalbac, M. Zukalova, L. Kavan, Probing highpressure properties of single-wall carbon nanotubes through fullerene encapsulation, Phys. Rev. B 77 (12) (2008) 125418. doi:10.1103/PhysRevB.77.125418.

[13] B. Anis, K. Haubner, F. Börrnert, L. Dunsch, M. H. Rümmeli, C. A. Kuntscher, Stabilization of carbon nanotubes by filling with inner tubes: An optical spectroscopy study on double-walled carbon nanotubes under hydrostatic pressure, Phys. Rev. B 86 (2012) 155454. doi:10.1103/PhysRevB.86.155454.

[14] A. Torres-Dias, S. Cambré, W. Wenseleers, D. Machon, A. San-Miguel, Chirality-dependent mechanical response of empty and water-filled single-wall carbon nanotubes at high pressure, Carbon 95 (2015) 442451. doi:10.1016/j.carbon.2015.08.032.

[15] D. Christofilos, J. Arvanitidis, E. Efthimiopoulos, X. Zhao, Y. Ando, T. Takenobu, Y. Iwasa, H. Kataura, S. Ves, G. A. Kourouklis, Tube encapsulation effects in various carbon nanotube systems, Phys. Status Solidi B 244 (11) (2007) 4082-4085. doi:10.1002/pssb.200776153. 
[16] A. Merlen, P. Toulemonde, N. Bendiab, A. Aouizerat, J. Sauvajol, G. Montagnac, H. Cardon, P. Petit, A. San Miguel, Raman spectroscopy of open-ended single wall carbon nanotubes under pressure: effect of the pressure transmitting medium, Phys. Status Solidi B 243 (3) (2006) 690-699. doi:10.1002/pssb.200541364.

[17] A. J. Ghandour, D. J. Dunstan, A. Sapelkin, G-mode behaviour of closed ended single wall carbon nanotubes under pressure, Phys. Status Solidi B 246 (3) (2009) 491-495. doi:10.1002/pssb.200880503.

[18] Y. Huang, J. Wu, K. C. Hwang, Thickness of graphene and single-wall carbon nanotubes, Phys. Rev. B 74 (2006) 245413. doi:10.1103/PhysRevB.74.245413.

[19] P. Lambin, Elastic properties and stability of physisorbed graphene, Appl. Sci. 4 (2) (2014) 282-304. doi:10.3390/app4020282.

[20] R. Nicklow, N. Wakabayashi, H. G. Smith, Lattice dynamics of pyrolytic graphite, Phys. Rev. B 5 (1972) 4951-4962. doi:10.1103/PhysRevB.5.4951.

[21] N. Lindahl, D. Midtvedt, J. Svensson, O. A. Nerushev, N. Lindvall, A. Isacsson, E. E. B. Campbell, Determination of the bending rigidity of graphene via electrostatic actuation of buckled membranes, Nano Lett. 12 (7) (2012) 3526-3531. doi:10.1021/nl301080v. 
[22] M. K. Blees, A. W. Barnard, P. A. Rose, S. P. Roberts, K. L. McGill, P. Y. Huang, A. R. Ruyack, J. W. Kevek, B. Kobrin, D. A. Muller, P. L. McEuen, Graphene kirigami, Nature 524 (7564) (2015) 204-207. doi:10.1038/nature14588.

[23] W. Wenseleers, S. Cambré, J. Čulin, A. Bouwen, E. Goovaerts, Effect of water filling on the electronic and vibrational resonances of carbon nanotubes: Characterizing tube opening by raman spectroscopy, Adv. Mater. 19 (17) (2007) 2274-2278. doi:10.1002/adma.200700773.

[24] S. Cambré, B. Schoeters, S. Luyckx, E. Goovaerts, W. Wenseleers, Experimental observation of single-file water filling of thin single-wall carbon nanotubes down to chiral index (5,3), Phys. Rev. Lett. 104 (20) (2010) 207401. doi:10.1103/PhysRevLett.104.207401.

[25] V. Vassilev, P. Djondjorov, I. Mladenov, Comment on "shape transition of unstrained flattest single-walled carbon nanotubes under pressure" [j. appl. phys. 115, 044512 (2014)], J. Appl. Phys. 117 (2015) 196101. doi:10.1063/1.4921233.

[26] J. Zang, A. Treibergs, Y. Han, F. Liu, Geometric constant defining shape transitions of carbon nanotubes under pressure, Phys. Rev. Lett. 92 (2004) 105501. doi:10.1103/PhysRevLett.92.105501.

[27] W. Yang, R. Z. Wang, X. M. Song, B. Wang, H. Yan, Pressure-induced raman-active radial breathing mode transition in 
single-wall carbon nanotubes, Phys. Rev. B 75 (2007) 045425. doi:10.1103/PhysRevB.75.045425.

[28] K. M. Liew, Y. Sun, Elastic properties and pressure-induced structural transitions of single-walled carbon nanotubes, Phys. Rev. B 77 (2008) 205437. doi:10.1103/PhysRevB.77.205437.

[29] Y. Sun, D. Dunstan, M. Hartmann, D. Holec, Nanomechanics of carbon nanotubes, Proc. Appl. Math. Mech. 13 (2013) 7-10. doi:10.1002/pamm.201310003.

[30] D. Holec, M. A. Hartmann, F. D. Fischer, F. G. Rammerstorfer, P. H. Mayrhofer, O. Paris, Curvature-induced excess surface energy of fullerenes: Density functional theory and Monte Carlo simulations, Phys. Rev. B 81 (2010) 235403. doi:10.1103/PhysRevB.81.235403.

[31] M. A. Hartmann, M. Todt, F. G. Rammerstorfer, F. D. Fischer, O. Paris, Elastic properties of graphene obtained by computational mechanical tests, Europhys. Lett. 103 (2013) 68004. doi:10.1209/0295$5075 / 103 / 68004$.

[32] T. Hayashi, Y. A. Kim, T. Matoba, M. Esaka, K. Nishimura, T. Tsukada, M. Endo, M. S. Dresselhaus, Smallest freestanding single-walled carbon nanotube, Nano Lett. 3 (7) (2003) 887-889. doi:10.1021/nl034080r. 
[33] N. M. Pugno, The design of self-collapsed super-strong nanotube bundles, J. Mech. Phys. Solids 58 (9) (2010) 1397-1410. doi:10.1016/j.jmps.2010.05.007.

[34] N. M. Pugno, J. A. Elliott, Buckling of peapods, fullerenes and nanotubes, Phys. E 44 (6) (2012) 944-948. doi:10.1016/j.physe.2011.12.024.

[35] L. Guan, K. Suenaga, S. Iijima, Smallest carbon nanotube assigned with atomic resolution accuracy, Nano Lett. 8 (2) (2008) 459-462. doi:10.1021/nl072396j.

[36] A. L. Aguiar, E. B. Barros, R. B. Capaz, A. G. Souza Filho, P. T. C. Freire, J. Mendes Filho, D. Machon, C. Caillier, Y. A. Kim, H. Muramatsu, M. Endo, A. San-Miguel, Pressure-induced collapse in double-walled carbon nanotubes: Chemical and mechanical screening effects, J. Phys. Chem. C 115 (13) (2011) 5378-5384. doi:10.1021/jp110675e.

[37] J. Singer, C. D. Babcock, On the buckling of rings under constant directional and centrally directed pressure, J. Appl. Mech 37 (1) (1970) 215-218. doi:10.1115/1.3408445.

[38] W. Cui, T. F. T. Cerqueira, S. Botti, M. A. L. Marques, A. SanMiguel, Nanostructured water and carbon dioxide inside collapsing 
carbon nanotubes at high pressure, Phys. Chem. Chem. Phys. 18 (2016) 19926-19932. doi:10.1039/C6CP03263J. 


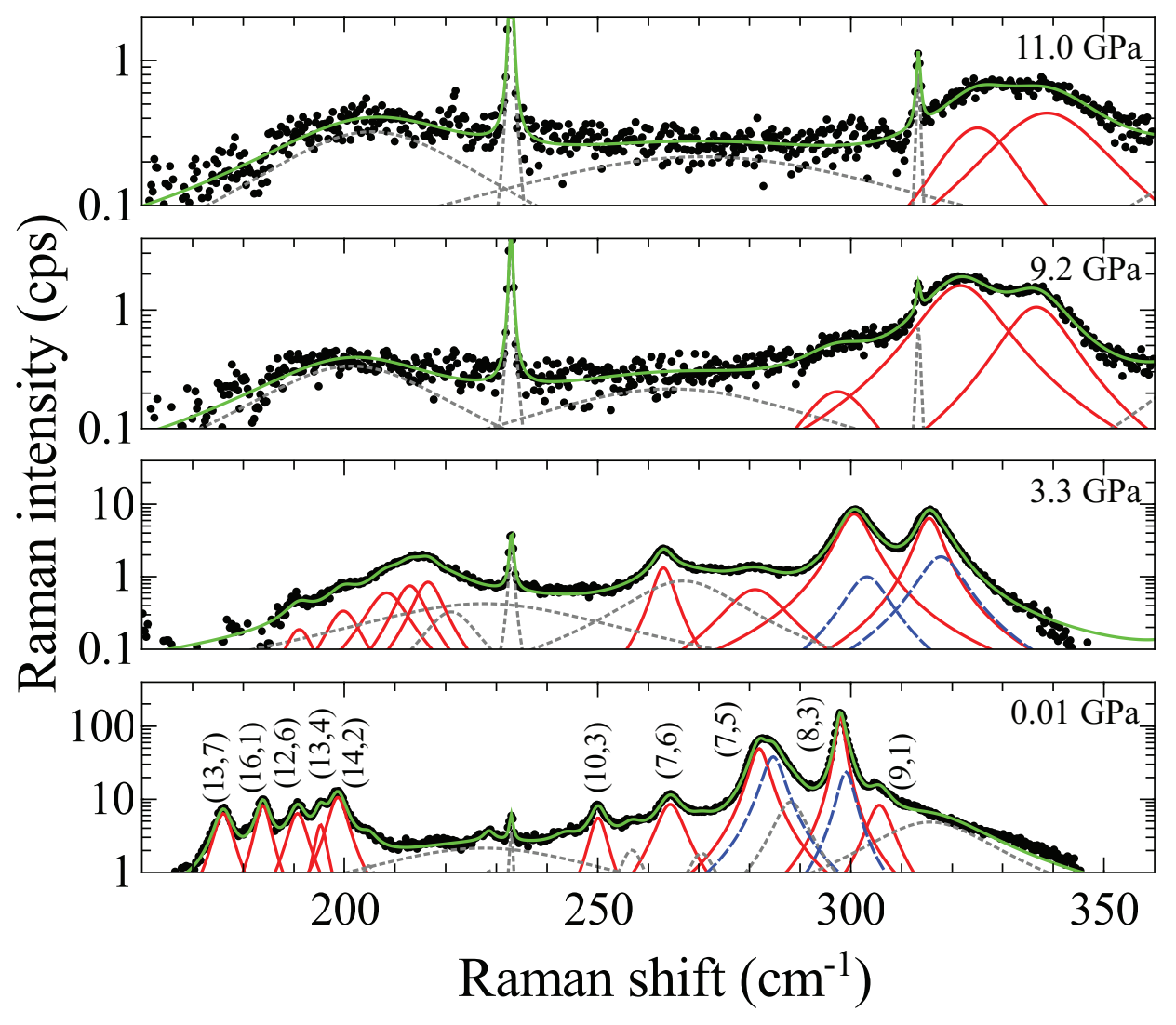

Figure 1: Raman spectra of radial breathing modes (RBMs) at different pressures, as semi-logarithmic plots of the spectra with their fits. The ten Lorentzians for the identified empty RBM peaks are shown by red solid lines; the blue dashed lines (water-filled RBMs) and the grey dotted lines show the other peaks. The solid green line shows the sum of all the fitted peaks, for comparison with the data (black dots). The excitation wavelength is $647.1 \mathrm{~nm}$. 


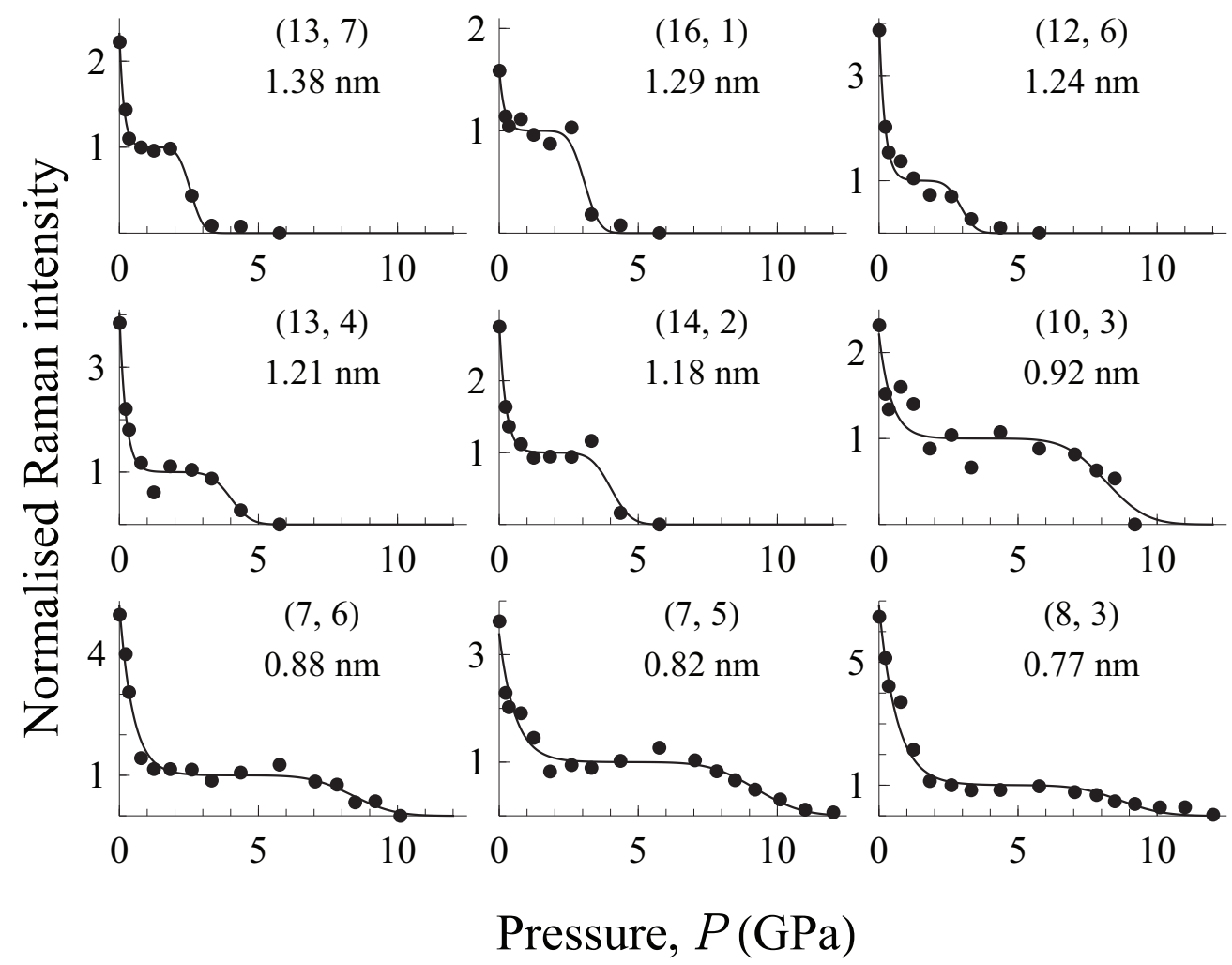

Figure 2: Outcomes of fitting the spectra of Figure 1. The normalised intensities of nine $(n, m) R B M$ peaks are plotted as a function of pressure. For each RBM, chiral indexes and diameter are shown on the graph

- The solid lines represent the fits with the function described in the text.

Data and fits have been normalized for a plateau value of 1 . 


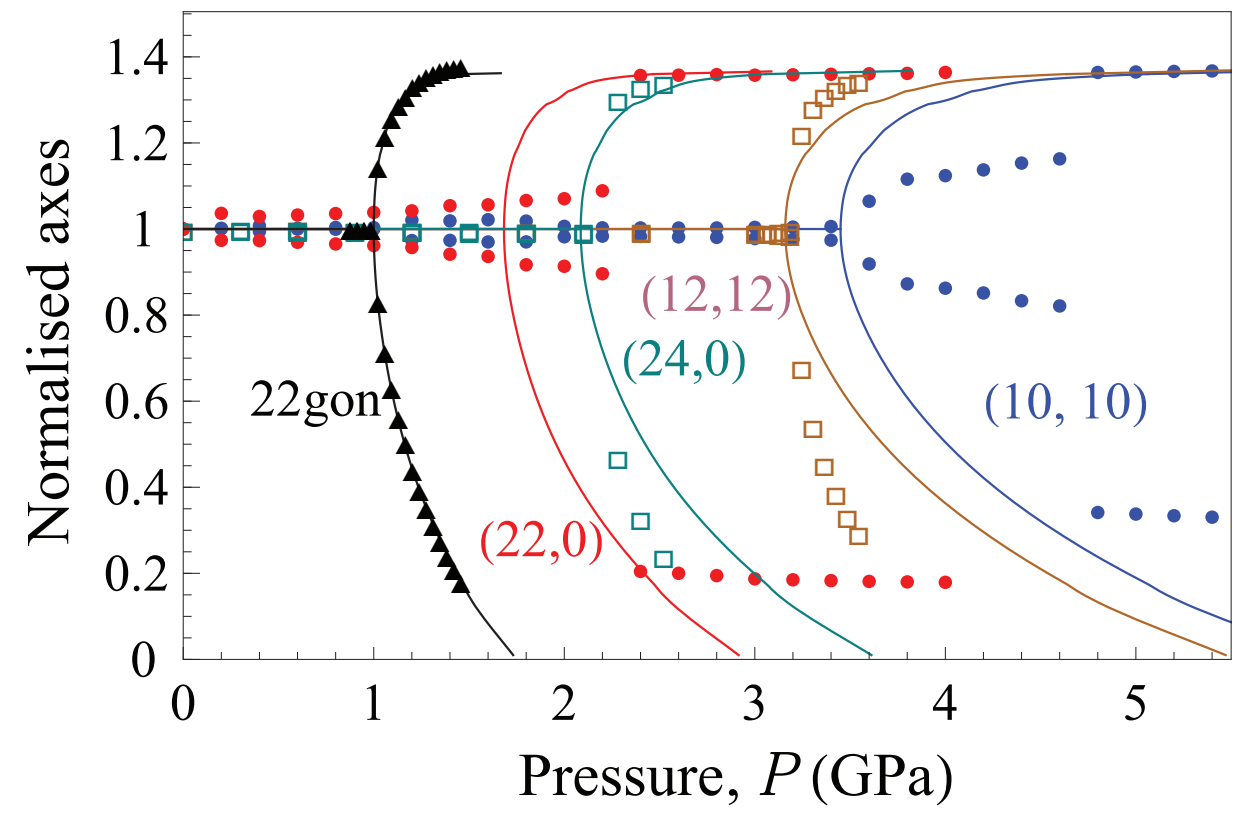

Figure 3: Calculated distortion of nanotubes during collapse, as the major and minor radial axes normalised to the nanotube diameter. The open squares are for $\mathrm{MC}$ calculations for $(24,0)$ and $(12,12)$ nanotubes. The solid circles are from DFTB of $(22,0)$ nanotubes in argon and $(10,10)$ nanotubes in water. The triangles are for a 22-gonal Hooke's Law atomistic ring with an arbitrary spring constant. The solid lines are predictions using the simple elastic ring with $D$ varied to fit each dataset. 


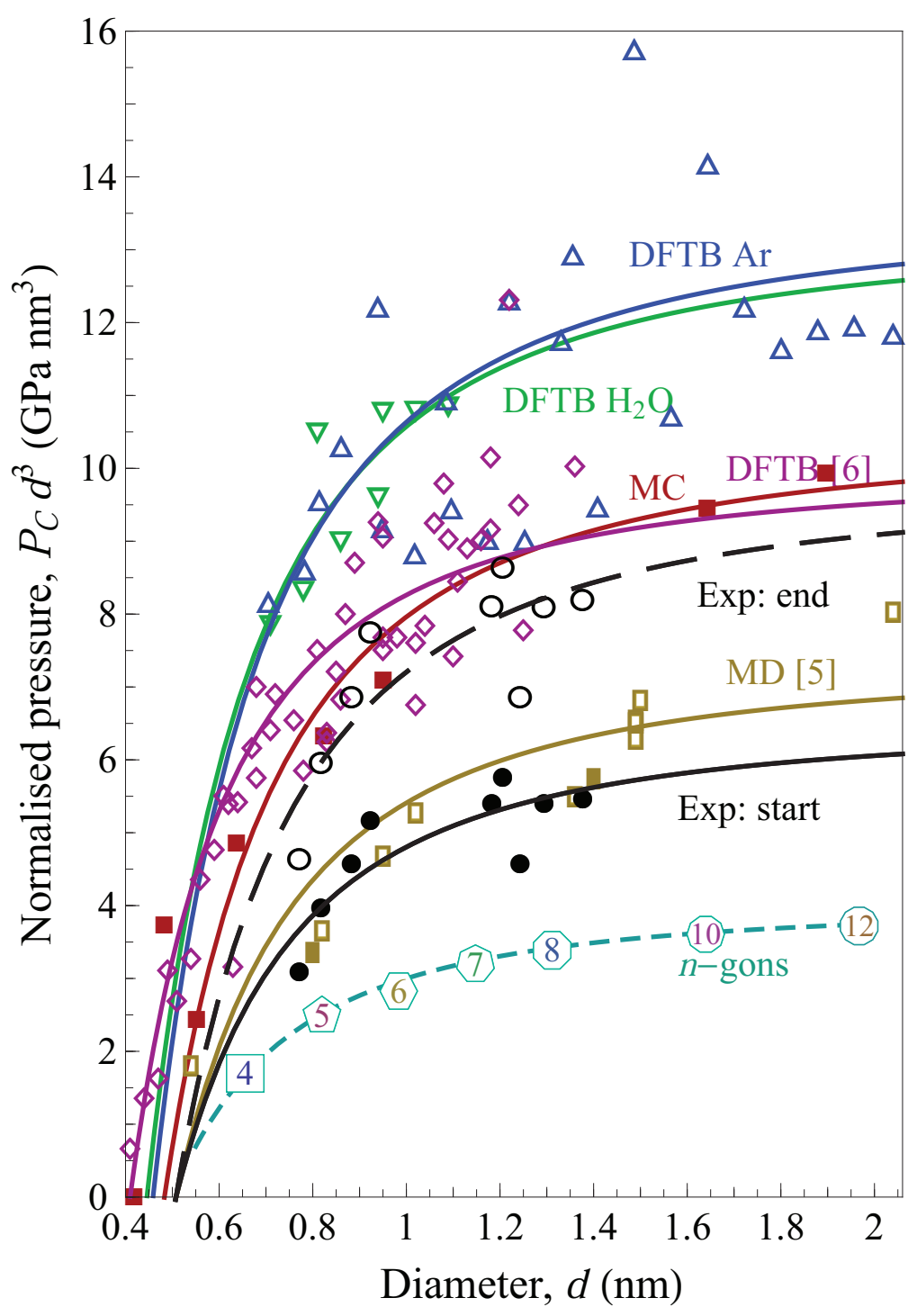

Figure 4: The experimental collapse pressures are compared with theoretical results in the form $P_{C} d^{3}$ plotted against $d$. In this plot, the simple elastic ring with bending modulus $D$ gives a horizontal line at $P_{C} d^{3}=24 D$ for the onset of collapse and at $\sim 36 D$ for the end of collapse. The lines are fits to the data using $P_{C} d^{3}=24 \alpha D\left(1-\beta^{2} / d^{2}\right)$ in which $\alpha$ is 1 for the onset of collapse and 1.5 for the end (see text). The dotted curve is the fit to the polygon-model results, which have been rescaled both in radius and in spring constant so that the shape may be compared with the other data. 

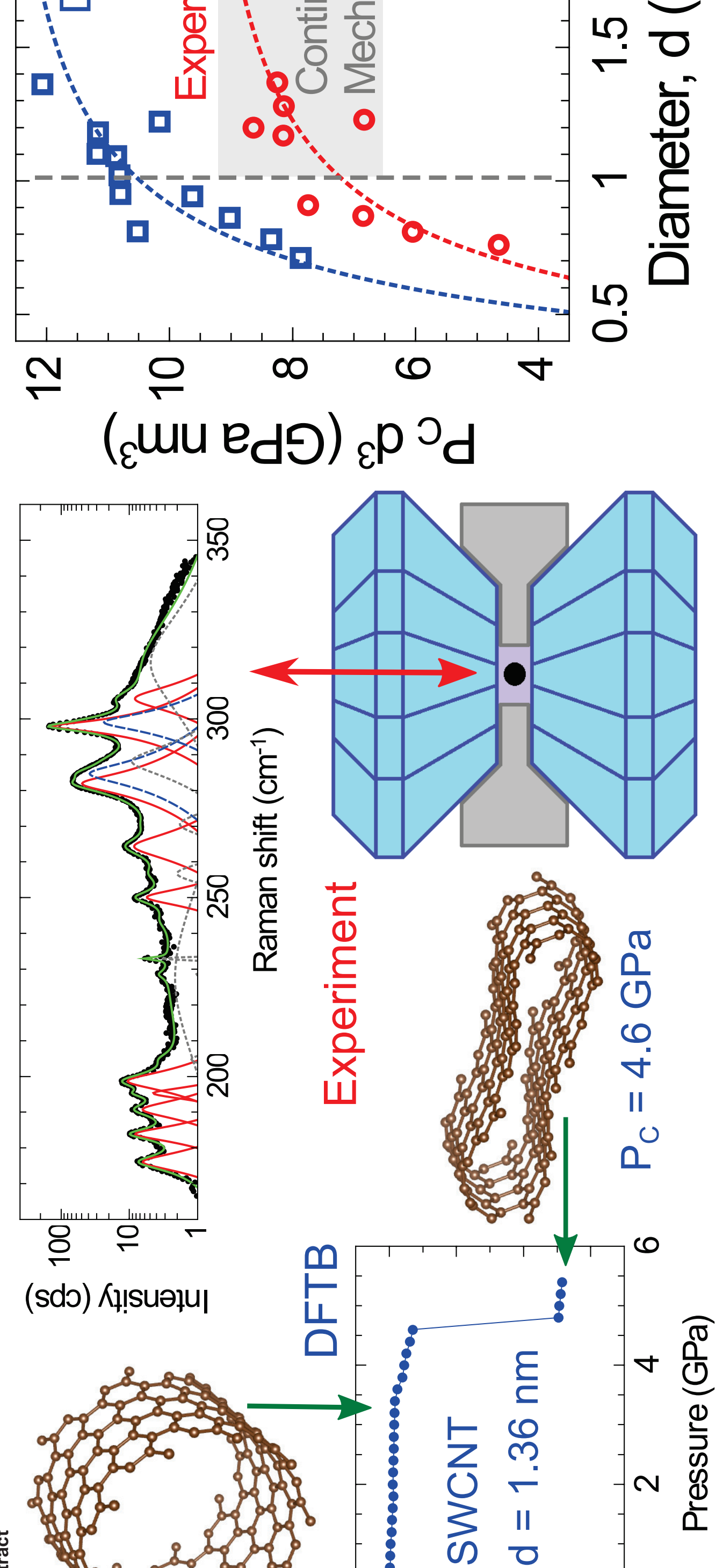